\title{
Population dynamics and management of culture-based fisheries
}

\section{K. LORENZEN}

Renewable Resources Assessment Group, Imperial College of Science, Technology and Medicine, University of London, United Kingdom

Abstract The population dynamics of culture-based fisheries are studied by means of a mathematical model, which incorporates explicit sub-models for density-dependent individual growth and size-dependent mortality. The model applies to populations of carps, i.e. common carp, Chinese carps, and Indian major carps, and coregonids.

The effects on production of stocking density and size of seed fish, fishing mortality, and size at harvesting are studied in a model population of carp. Management implications of the modelling results are emphasized. An adaptive approach to management, involving judicious experimentation with stocking and harvesting regimes, is proposed to gain information on the dynamics of actual fisheries, and to optimize their production.

KEYWORDS: Aquaculture, assessment, enhancement, management, model, stocking.

\section{Introduction}

Culture-based fisheries are capture fisheries which are mostly or entirely maintained by the regular stocking of seed fish. Culture-based fisheries rely entirely on the natural productivity of the water body for growth, and on artificial stocking for recruitment.

Commercial culture-based inland fisheries are operated on a large scale in Asia, particularly in China (De Silva, Yu \& Xiang 1991), and on the Indian Subcontinent (Jhingran 1992). The main species stocked in Asia are Chinese carps, Indian major carps, and common carp, Cyprinus carpio L. Many European lakes are also regularly stocked with coregonids to support commercial fisheries (FAO 1994). Major culture-based fisheries, both commercial and recreational, also exist for salmonids. This study investigates the population dynamics of culture-based carp fisheries, as are typically found in the Asian region.

Culture-based fisheries pose assessment and management problems which are difficult to address using established methodology for natural stocks. This theoretical study investigates some aspects of the population dynamics of culture-based fisheries, derives management guidelines and outlines an adaptive approach to the management of such fisheries.

The model is intended to provide general insights, and uses some simplifying assumptions concerning growth, mortality and the management regime. When the 
model is applied to an actual fishery, the validity of these assumptions and the consistency of model predictions with available data should be checked carefully.

\section{Material and methods}

This study refers to a culture-based carp fishery in a perennial water body, as commonly operated in many Asian reservoirs. For simplicity, it is assumed that the fishery is stocked and harvested continuously throughout the year. Most fisheries are stocked once a year and harvested continuously, but this does not affect the conclusions drawn from this theoretical study.

\section{The population model}

The dynamics of the stocked fish population are determined by two processes, individual growth and mortality. Because the fish depend entirely on the natural food supply, individuals compete for a limited food resource, and consequently their growth is dependent on population density. Growth in the model population is described by a density-dependent extension of the von Bertalanffy growth function (VBGF), where the asymptotic length $L_{\infty}$ is a linear decreasing function of population biomass:

$$
\mathrm{L}_{x B}=\mathrm{L}_{{ }_{x L}}-d B
$$

In this model, $L_{\infty B}$ is the asymptotic length of the fish growth curve at biomass $\mathrm{B}$, and $L_{\infty L}$ is the limiting asymptotic length which a fish would approach in the absence of competition. Even in the absence of competition, the asymptotic length of fish is limited by the natural productivity of the water body, which is thus reflected in $L_{\infty L L}$. The competition coefficient $\mathrm{d}$ is the amount by which $L_{\infty B}$ decreases per unit of population biomass. Biomass refers to standing crop, i.e. biomass per unit area. The growth model is explained in more detail, and applied to the analysis of extensive carp culture experiments, in Lorenzen (1993).

The mortality rate in the model population is size dependent: small fish suffer a higher mortality rate than large ones. The mortality-size relationship is described by the following model, first proposed by Gulland (1987):

$$
M_{L}=M_{r}\left(L / L_{r}\right)^{-3 \rho}
$$

where $M_{L}$ is the mortality rate at body length $\mathrm{L}$, and $M_{r}$ is the mortality rate at reference length $L_{r}$ The exponent of the mortality-length relationship is $-3 \rho$, and $-\rho$ is the exponent of the corresponding mortality- weight relationship for isometric growth. The representation of the mortality-size relationship as a power function was derived theoretically by Peterson \& Wroblewski (1984), and there is substantial empirical evidence for this form of the relationship in the marine pelagic ecosystem (McGurk 1986, 1987). Gulland (1987) introduced the above model, where the coefficient $M_{r}$ is equal to the natural mortality at a conveniently chosen reference length $L_{r}$.

The models for density-dependent growth and size-dependent mortality are combined in a length-structured population model. The fish population is divided into length 


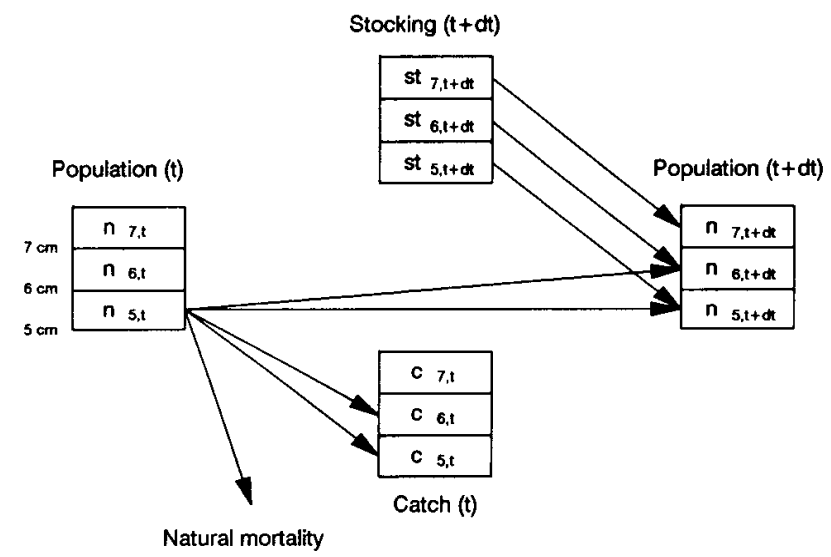

Figure 1. Flow chart of the length-structured population model. Arrows indicate the transition of fish between length groups of the population over time, from the population to catch and natural death, and from stocking to the population.

groups, and the model projects population and catch numbers-at-length over time (Fig. 1). Fish in the $5 \mathrm{~cm}$ group at time $t$ will, during time interval $d t$, either stay in the population and grow, get caught in the fishery, or die naturally. The proportions of fish remaining in the population, being caught, or dying naturally are different in each length group, due to the size-dependence of both natural and fishing mortality. In the $5 \mathrm{~cm}$ group, for instance, a high proportion of individuals dies from natural causes, but none from fishing. At $7 \mathrm{~cm}$, fewer fish die naturally. In the larger length groups, e.g. at $30 \mathrm{~cm}$, very few fish die naturally, but most may be harvested. During the time interval $d t$, fish will also grow and some of the individuals from the $5 \mathrm{em}$ group will move to the $6 \mathrm{~cm}$ group, while others will remain in the $5 \mathrm{~cm}$ group. The same applies to the fish harvested. The proportions of fish remaining in a length group or growing into another vary between length groups, and with population biomass. Population numbers-at-length are increased by the numbers stocked-at-length at the beginning of each time step).

The complete mathematical formulation of the population model is given in the Appendix.

\section{Parameter values}

The parameter values of the model fish population were chosen to represent a hypothetical, culture-based carp fishery (Table 1). In the growth model, the limiting asymptotic length is set to $80 \mathrm{~cm}$. In conjunction with a competition coefficient $d$ of $0.5 \mathrm{~cm} \mathrm{ha}{ }^{-1} \mathrm{~kg}^{-1}$, fish will approach an $L_{\infty B}$ of $70 \mathrm{~cm}$ at a population biomass of $20 \mathrm{~kg} \mathrm{ha}^{-1}$ or an $L_{\infty B}$ of $30 \mathrm{~cm}$ if the population biomass is $100 \mathrm{~kg} \mathrm{ha}^{-1}$. The parameter $\mathrm{K}$ is set to $0.4 \mathrm{y}^{-1}$.

In the mortality-size relationship, the exponent $\rho$ is assigned a value of 0.5 . Empirical studies have found $\rho$ to be 0.44 in the marine pelagic ecosystem (McGurk 1986, 1987), and between 0.36 and 0.98 in European carp pond culture (Lorenzen 
Table 1. Parameter values of the model population.

\begin{tabular}{ll}
\hline Parameter & \multicolumn{1}{c}{ Value } \\
\hline Density-dependent VBGF' & \\
$L_{\times L}$ & $80 \mathrm{~cm}$ \\
$\mathrm{~K}$ & $0.4 \mathrm{y}^{-1}$ \\
$d$ & $0.5 \mathrm{~cm} \mathrm{ha} \mathrm{kg-1}^{-1}$ \\
Size-dependent mortality & \\
$\rho$ & 0.5 \\
$L_{r}$ & $20 \mathrm{~cm}^{-1}$ \\
$M_{r}$ & $0.6 \mathrm{y}^{-1}$ \\
Weight-length relationship & \\
$a$ & $2.5 \times 10^{-5} \mathrm{~kg} \mathrm{~cm}^{3}$ \\
$b$ & 3.0 \\
\hline
\end{tabular}

1993). The mortality rate $M$, is $0.6 y^{-1}$ at a reference length $L_{r}$ of $20 \mathrm{~cm}$. This corresponds to mortality rates of $1.7 \mathrm{y}^{-1}$ at $10 \mathrm{~cm}$, and $4.8 \mathrm{y}^{-1}$ at $5 \mathrm{~cm}$.

The length -weight relationship is isometric $(b=3.0)$, with a coefficient $\mathrm{a}=2.5 \times 10-5 \mathrm{~kg}$ $\mathrm{cm}^{-3}$, so that a $20 \mathrm{~cm}$ long fish weighs $0.2 \mathrm{~kg}$.

The length of seed fish is assumed to be normally distributed, with a standard deviation of $1 \mathrm{~cm}$. The mean length is $5 \mathrm{~cm}$, and is later varied between $3 \mathrm{~cm}$ and $11 \mathrm{~cm}$ to explore the effect of seed size on production.

Knife-edge gear selection is assumed at selection length 1, i.e. all fish larger than 1, are retained by the gear, while all smaller fish can escape. The selection length $1_{\mathfrak{c}}$, is set to $30 \mathrm{~cm}$, and is later varied between $25 \mathrm{~cm}$ and $40 \mathrm{~cm}$.

As described earlier, both stocking and harvesting are continuous throughout the year, and at a constant rate. The fish population is then in a state of dynamic equilibrium, i.e. its size structure and biomass remain constant over time.

\section{Results}

The response of the model population to various stocking and harvesting regimes is demonstrated in the following sections. The results are presented in separate sections for stocking density, size at harvesting and fishing mortality in relation to stocking density, and the size of seed fish.

\section{Stocking density}

The influence of stocking density on production and the recapture rate of stocked fish is illustrated in Fig. 2. The mean length of seed fish is constant at $5 \mathrm{~cm}$. Gear selection length 1, is $30 \mathrm{~cm}$, and fishing mortality is taken to be very high, so that fish are harvested immediately after reaching $1_{c}$ The average weight of fish in the catch is about $0.7 \mathrm{~kg}$.

The biomass stocked always increases proportionally to stocking density in numbers, 


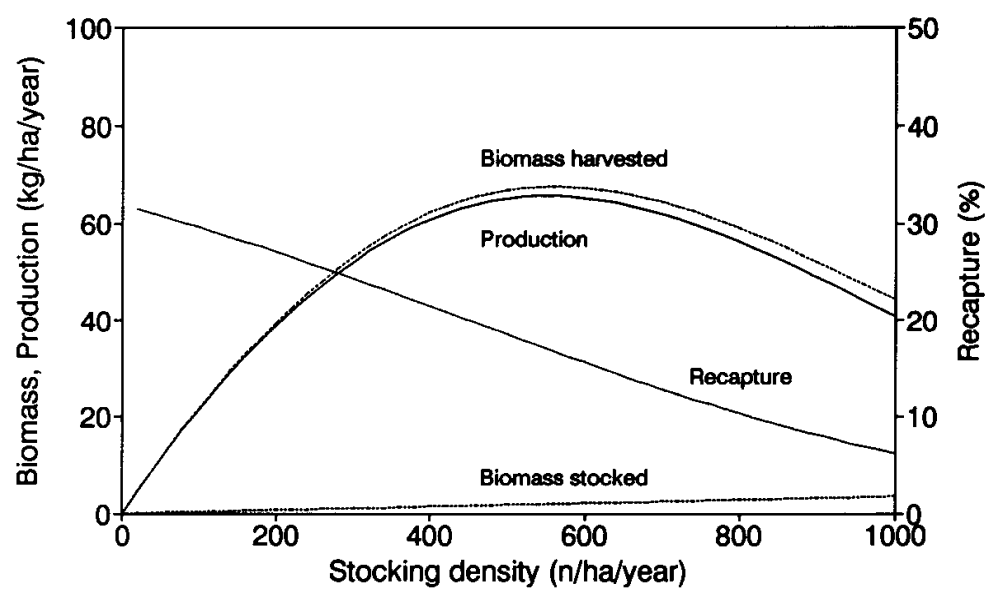

Figure 2. Stocked biomass, harvested biomass, and production as a function of stocking density in numbers. Also shown is the fraction of seed fish recaptured in the fishery. Standard parameters values (Table 1), gear selection length $1_{\mathrm{c}}=30 \mathrm{~cm}$, and very high fishing mortality.

because the mean weight of seed fish is constant at about $3.5 \mathrm{~g}$ per individual. The biomass harvested increases almost proportionally to stocking density at low densities, but the rate of increase declines as stocking density increases. This reflects the density-dependent reduction in individual growth, and the consequent increase in natural mortality. The maximal biomass harvested is $67.6 \mathrm{~kg} \mathrm{ha}^{-1} \mathrm{y}^{-1}$, at a stocking density of 560 individuals ha ${ }^{-1} \mathrm{y}^{-1}$. The biological production of the fishery is equal to the biomass harvested minus the biomass stocked. Production reaches a maximum when the slope of the 'biomass harvested' curve is equal to that of the 'biomass stocked' line, at a stocking density slightly lower than that at which the maximal biomass is harvested. If stocking density is increased beyond 560 individuals ha ${ }^{-1} \mathrm{y}^{-1}$, this results in a decline of both biomass harvested and production, i.e. the fishery is overstocked. At high population densities, fish grow slowly and consequently suffer higher natural mortality. This is apparent in the fraction of stocked fish which are recaptured in the fishery. At very low stocking densities, the recapture rate is highest (about 30\%) due to the fast growth of individuals. The recapture rate is much lower (about 15\%) in the region of optimal stocking density, and declines further when the fishery is overstocked. Clearly, the return per seed fish stocked is highest at low stocking density. However, the overall production optimum is reached when the recapture rate and the consequent return per seed fish are at an intermediate level.

\section{Size at harvesting}

The optimal stocking density in a culture-based fishery is dependent on the size of the fish harvested. In Fig. 3, production is shown as a function of stocking density for gear selection lengths 1 , of 25, 30, 35 and $40 \mathrm{~cm}$, corresponding to weights of about $0.4,0.7,1.1$ and $1.7 \mathrm{~kg}$ respectively. Fishing mortality is taken to be very high, so that fish are 


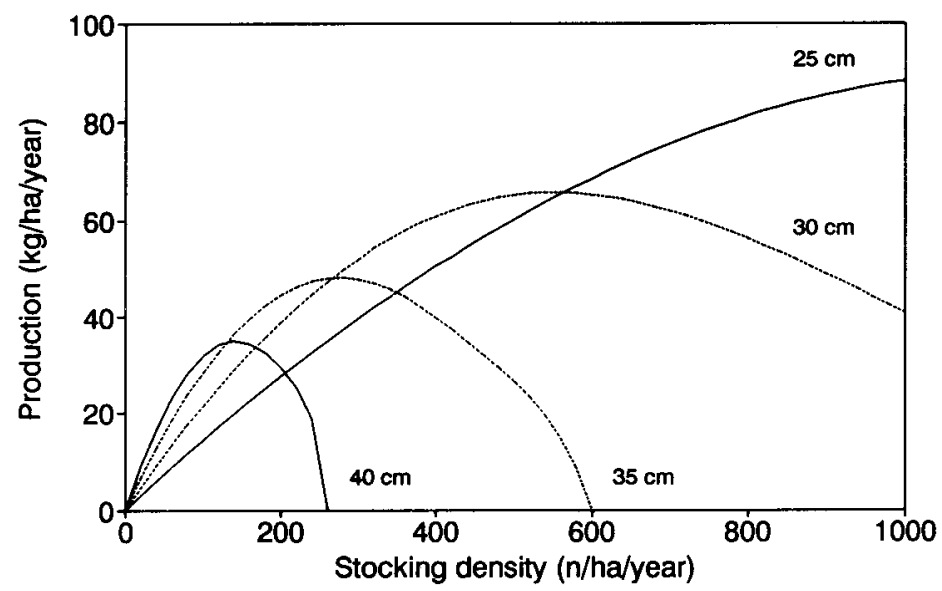

Figure 3. Production as a function of stocking density, for four different gear selection lengths $1_{\mathrm{c}}$ between 25 and $40 \mathrm{~cm}$. Standard parameter values (Table 1) and very high fishing mortality.

caught immediately after reaching $1_{\mathrm{c}}$ and the corresponding weight. The mean length of seed fish is constant at $5 \mathrm{~cm}$.

At an $1_{\mathrm{c}}$ of $40 \mathrm{~cm}$, the maximal production of $35 \mathrm{~kg} \mathrm{ha}^{-1} \mathrm{y}^{-1}$ is reached at a low stocking density of only 140 individuals ha $\mathrm{y}^{-1} \mathrm{y}^{-1}$. If stocking density is increased beyond this optimum, production declines rapidly and drops to zero before 300 individuals $\mathrm{ha}^{-1} \mathrm{y}^{-1}$ are reached. Moderate overstocking results in sub-optimal production due to slow growth and high mortality, but the fishery can still operate. If stocking density is increased further, the asymptotic length $L_{\propto \circ B}$ of the population will fall below the gear selection length $1_{\mathrm{c}}$. This brings about a qualitative change as catches drop to zero, and the fishery no longer removes biomass from the population. If stocking continues, the water body is 'choked' with fish, resulting in a dense, stunted population. At a high gear selection length such as $40 \mathrm{~cm}$, the critical biomass is reached easily, and overstocking can be a serious management problem.

The maximal production at $1_{\mathrm{c}}=35 \mathrm{~cm}$ is $48 \mathrm{~kg} \mathrm{ha}^{-1} \mathrm{y}^{-1}$ at a stocking density of 280 individuals $\mathrm{ha}^{-1} \mathrm{y}^{-1}$. Obviously, the potential production increases with decreasing size at harvesting, but this production is achieved only at increasingly high stocking densities. For the harvesting sizes considered in this example, the overall production maximum is at $1_{c}=25$ $\mathrm{cm}$, at a stocking density of over 1000 individuals $\mathrm{ha}^{-1} \mathrm{y}^{-1}$. Unless stocking densities are limited by the availability of seed, it is more productive to harvest fish at the smallest marketable size.

When stocking density is limited by seed supply, this also dictates the optimal harvesting size. For example, if stocking density is limited to 500 individuals $\mathrm{ha}^{-1} \mathrm{y}^{-1}$, the optimal size for harvesting is about $30 \mathrm{~cm}(0.7 \mathrm{~kg})$ (Fig. 3). In general, if stocking density is limited, the optimal size for harvesting may be much larger than the smallest marketable size.

If only large fish are marketable, it is counter-productive to stock at high densities. For example, if the smallest marketable size for catches from the model population was 


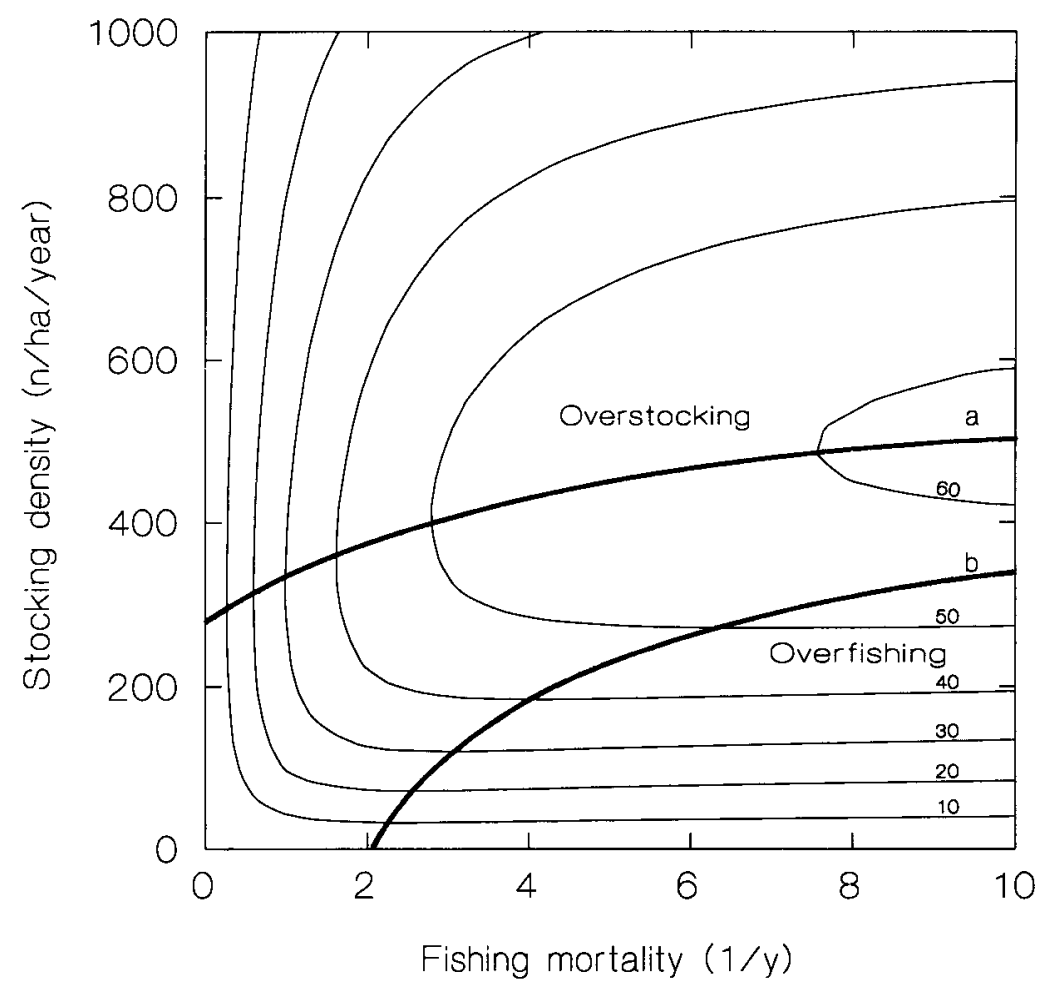

Figure 4. Production as a function of fishing mortality and stocking density, for a gear selection length $1_{\mathrm{c}}$ of $30 \mathrm{~cm}$. Labels of the contour lines indicate production in $\mathrm{kg} \mathrm{ha}^{-1} \mathrm{y}^{-1}$. The heavy solid line (a) indicates optimal stocking density in relation to fishing mortality, line (b) indicates optimal fishing mortality in relation to stocking density. Standard parameter values (Table 1).

1. $1 \mathrm{~kg}(35 \mathrm{~cm})$, no more than 280 seed fish of $5 \mathrm{~cm}$ length should be stocked per ha annually.

\section{Fishing mortality}

The problem of fishing mortality is linked to the size at harvesting: the higher the fishing mortality for a set gear selection length 1, the lower the average size of harvested fish. This implies that a high fishing mortality calls for a high stocking density, and vice versa.

The combined effect of fishing mortality and stocking density on production is illustrated in Fig. 4, for a constant $1_{\mathrm{c}}$ of $30 \mathrm{~cm}$, and a mean size of seed fish of $5 \mathrm{~cm}$. Production contour lines indicate the combinations of fishing mortality and stocking density that give rise to the same levels of production. For example, a production of $40 \mathrm{~kg} \mathrm{ha}^{-1} \mathrm{y}^{-1}$ can be achieved by stocking 200 fish ha ${ }^{-1} \mathrm{y}^{-1}$ and fishing at $F=2.5 \mathrm{y}^{-1}$, or by stocking $600 \mathrm{fish} \mathrm{ha}^{-1} \mathrm{y}^{-1}$ and fishing at just above $F=2.0 \mathrm{y}^{-1}$. The heavy solid line (a) shows optimal stocking density in relation to fishing mortality. If stocking densities are above this line the fishery is overstocked, and a reduction in stocking 


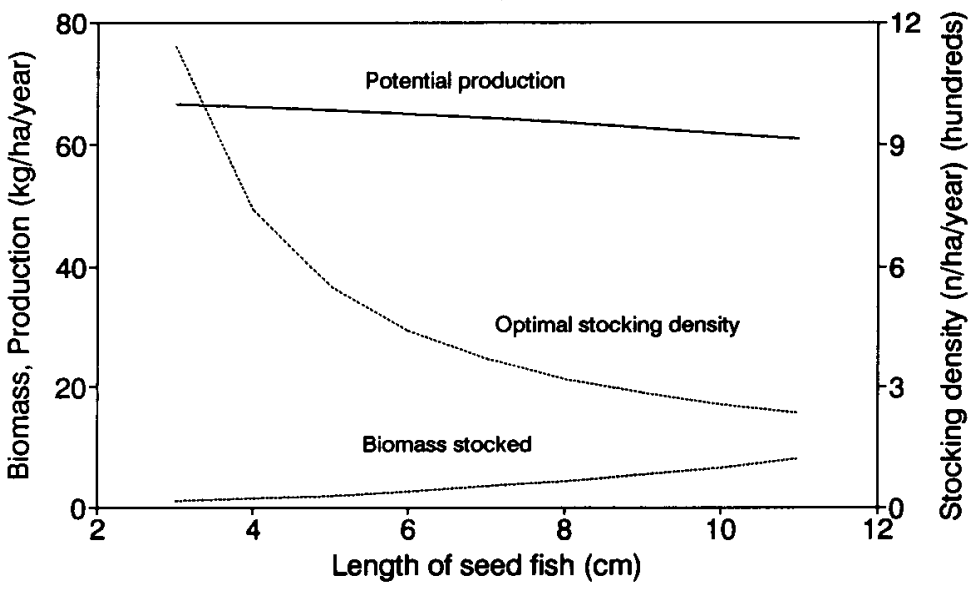

Figure 5. Influence of the length of seed fish on potential production, optimal stocking density and the corresponding biomass stocked. Optimal stocking density is the density required to realize potential production. Standard parameter values (Table 1), gear selection length $1_{\mathrm{c}}=30 \mathrm{~cm}$, and very high fishing mortality.

density will increase production. The same effect can be achieved by an increase in fishing mortality. The heavy solid line (b) shows the optimal fishing mortality in relation to stocking density. If the fishery is operated below this line, it is overfished and a reduction in fishing mortality will increase production. Alternatively, overfishing can be avoided by increasing stocking density. In this example, an increase in stocking density at fixed fishing mortality will improve production substantially, while a reduction in fishing mortality at fixed stocking density will have a more limited effect.

Both overstocking and overfishing can be averted by a change in either the stocking or the harvesting regime. The most suitable alternative depends on the current position of the fishery in its fishing mortality/stocking density plane.

As a rule, high stocking density requires high fishing mortality, and vice versa. In a developing culture-based fishery, it is important to increase both stocking density and fishing mortality in a balanced way.

\section{Size of seed fish}

Selecting the size of seed fish for stocking is a crucial aspect of the management of culture-based fisheries. The best recapture rate, and hence return per seed fish, is usually obtained by stocking large fish, because mortality is size-dependent. However, large seed fish are expensive to produce and their use also has other implications which are illustrated for the model population (Figs 5 and 6). The size of seed fish is varied between $3 \mathrm{~cm}$ and $11 \mathrm{~cm}$, while harvesting with a constant gear selection $\left(1_{c}=30 \mathrm{~cm}\right)$ and very high fishing mortality.

In Fig. 5, potential production is shown as a function of the length of seed fish, together with the stocking density and seed biomass required to achieve the potential production. Obviously, any size of seed fish within the range considered can yield a 


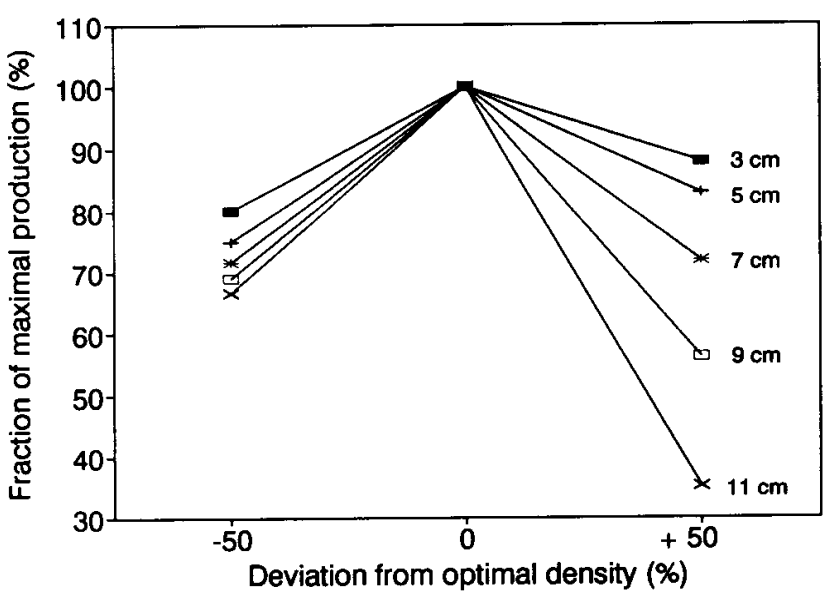

Figure 6. Sensitivity of production to sub-optimal stocking density, for different sizes of seed fish. The effect on production of a $50 \%$ deviation from optimal stocking density is shown. Standard parameter values (Table 1), gear selection length $1_{\mathrm{c}}$ $=30 \mathrm{~cm}$, and very high fishing mortality.

similar level of production. There is a slight decrease of potential production with increasing length of seed, which is explained later.

The optimal stocking density, at which potential production is obtained, declines sharply with the size of seed fish, from over $1100 \mathrm{ha}^{-1}$ for $3 \mathrm{~cm}$ to less than $300 \mathrm{ha}^{-1}$ for $11 \mathrm{~cm}$ fingerlings. The form of this curve reflects the mortality-size relationship, and the steepest decrease in optimal stocking density occurs in the low size range. Increasing the size of seed fish from 3 to $5 \mathrm{~cm}$ reduces the number of seed required by more than $50 \%$, while an increase in size from $9 \mathrm{~cm}$ to $11 \mathrm{~cm}$ reduces the numbers required by only $15 \%$. The best size for stocking is likely to be in the intermediate range, striking a balance between the need to produce vast numbers of individuals, and the need to rear them to a large size.

Also shown in Fig. 5 is the total biomass of seed stocked at the optimal density in numbers. Large seed fish must be stocked at a much higher biomass than small fish: while $1 \mathrm{~kg} \mathrm{ha}^{-1} \mathrm{y}^{-1}$ of $3 \mathrm{~cm}$ fish is sufficient to achieve maximal production, $11 \mathrm{~cm}$ fingerlings should be stocked at $9 \mathrm{~kg} \mathrm{ha}^{-1} \mathrm{y}^{-1}$. The use of large seed fish implies the necessity to produce and stock a relatively high biomass of seed. This fact also explains the slight decline in production, which is defined as biomass harvested minus biomass stocked, with increasing size of seed fish.

An important aspect related to optimal seed size is the sensitivity of production to stocking at non-optimal densities. This is illustrated in Fig. 6, which shows the changes in production for stocking densities $50 \%$ below and above the optimum, for different sizes of seed fish. For $3 \mathrm{~cm}$ fish, $50 \%$ understocking reduces production to $80 \%$ of the maximum, while $50 \%$ overstocking reduces production to $88 \%$. Production from $11 \mathrm{~cm}$ seed fish is much more sensitive; a $50 \%$ understocking reduces production to $66 \%$, but the same percentage overstocking causes a reduction to $35 \%$ of the maximal production.

Production from large seed fish is therefore more sensitive to stocking density than 
production from small seed. Hence, if the optimal density is not known from experience, stocking small seed fish is more likely to yield good results than stocking large seed fish. Production from small seed is most sensitive to understocking, while the production from large seed is particularly sensitive to overstocking.

\section{Discussion}

\section{The model}

The population model upon which this study is based can be applied to virtually any management regime, and it is not limited to the equilibrium situation considered here. The model can also be used for the estimation of parameters from stocking and catchat-length data.

The model is valid only for populations in which individual growth is density dependent, and the instantaneous mortality rate is size dependent but density independent. In addition, the growth model relies on the assumption that total biomass is a good predictor of competition effects on growth, and that all individuals are affected to the same degree. All these assumptions are likely to be met approximately by the cyprinids and coregonids stocked in Asia and Europe. Lorenzen (1993) has demonstrated the applicability of the growth and mortality models to extensive carp culture.

However, the model is not applicable to salmonid fisheries, another important group of culture-based fisheries. Young salmonids are often stocked in rivers or streams, where they suffer strongly density-dependent mortality while the growth of survivors is density independent (Elliott 1985, 1987). The validity of the model for predatory species in general is also doubtful. Predators usually undergo major ontogenetic changes in diet, so that different size-groups utilize different food resources and total biomass is a poor predictor of competition effects on growth.

The model is entirely deterministic, it does not take into account stochastic variability in parameter values. Such variability can be an important consideration, particularly with regard to the size of seed fish. Survival is often regarded as more variable for small than for large seed fish (FAO 1994). If this is the case, the stocking of small seed fish can result in highly variable production, although on average production is higher and less sensitive to stocking density than for large seed.

\section{Parameter estimation}

Relevant population parameters must be estimated to predict quantitatively the effects of management measures on a particular fishery. Some parameter values can be inferred from external information, while others can only be estimated from stocking and catch data for the particular fishery.

The parameter $\mathrm{K}$ in the density-dependent VBGF is the same as in the standard VBGF and can be inferred from comparative studies, but the parameters $L_{\propto} L$ and d must be estimated for each fishery separately. This is possible only if data on individual growth are available over a range of biomass densities. 
In the mortality-size relationship, a priori information on the value of $\rho$ can be gained from ecological theory (Peterson \& Wroblewski 1984), and empirical studies (McGurk 1986, 1987; Lorenzen 1993). The parameter $\mathrm{M}_{\mathrm{r}}$ should be estimated from stocking and catch data. If both parameters of the mortality-size relationship are to be estimated directly, data must be available over a range of seed fish sizes.

\section{Adaptive management}

Although the model parameters can be estimated as outlined above, their values will often be subject to a high degree of uncertainty. This particularly applies to newly established fisheries, or to fisheries with no record of stocking and catch data.

However, the management of culture-based fisheries under uncertainty need not be left to trial and error. Even under very high uncertainty, it is often possible to adopt management measures which are likely to simultaneously improve production and yield important information, i.e. reduce uncertainty. A management strategy which serves both objectives is called adaptive management (Walters 1986). Adaptive management entails judicious experimentation with stocking and harvesting regimes, and a systematic analysis of the outcome of such experiments.

A prerequisite for adaptive management is the collection of stocking and catch data, so that the present status of the fishery and the effects of any changes in management can be assessed. Stocking and catch data can be obtained easily from seed production centres and regular (not necessarily frequent) sampling at landing sites. It may also be useful to mark certain batches of fingerlings, e.g. by fin clipping. This is likely to improve the precision of growth-parameter estimates.

The following example illustrates the adaptive approach to management. Consider a fishery for which stocking and catch data are available for one production cycle (time between stocking and harvesting of a cohort). Growth and mortality parameters can be estimated from the data, and from empirical models. A yield-per-recruit analysis (Gulland 1983) will indicate whether the population is overfished or underfished. If the analysis indicates overfishing, an increase in stocking density will improve production. However, because the growth response to changes in density is not known, it is not possible to predict by how much the density should be increased. The choice of a new stocking density involves a trade-off between the gain of information and the risk of temporarily losing yield. A moderate increase in density will almost certainly increase yield, but it may not produce a significant growth response. A strong increase in density will produce a strong response and thus yield better information, but it may lead to overstocking and consequent loss of yield. If stocking density is increased sufficiently to produce a growth response, it is possible to obtain a first quantitative estimate of the optimal stocking density for a given level of fishing mortality. The predicted optimal stocking density is, of course, still subject to considerable uncertainty. Hence the effects of the new density must again he monitored and analysed.

Adaptive management is a continuous process: it is not finished once the current stocking and harvesting regimes appear to be optimal. Monitoring continues to detect changes in the ecology of the fishery, or in its exploitation. Moreover, management 
objectives are likely to change, for example in response to changing socio-economic factors. Adaptive management entails a systematic and efficient way of responding to such changes, thereby ensuring the sustainability of the culture-based fishery.

\section{Acknowledgements}

1 am grateful to Dr Sophie des Clers for advice and discussions during the course of this work. Professor John Beddington read and commented on the manuscript. This study was supported by the Overseas Development Administration of the British Government, under the Fishery Management Science Programme.

\section{References}

De Silva, S.S., Yu Z. \& Xiang L.H. (1991) A brief review of the status and practices of the reservoir fishery in mainland China. Aquaculture and Fisheries Management 22, 73-84.

Elliott LM. (1985) Population dynamics of migratory trout, Salmo trutta, and their implications for fisheries management. Journal of Fish Biology 27(Suppl. A), 35-43.

Elliott J.M. (1987) Population regulation in two contrasting populations of trout, Salmo trutta, in two Lake District streams. Journal of Animal Ecology 56, 83-98.

FAO (1994) Guidelines for Stocking Coregonids. FAO, EIFAC Technical Paper (in press).

Gulland, J.G. (1983) Fish Stock Assessment: A Manual of Basic Methods. Chichester: Wiley, 223 pp.

Gulland LG. (1987) Natural mortality and size. Marine Ecology - Progress Series 39, 197-199.

Jhingran A.G. (1992) Fisheries status of aquaculture-based open water systems in India. In: V.R.P. Sinha \& H.C. Srivastava (eds). Aquaculture Productivity. New Delhi: Oxford and IBH Publishing Co. pp. 295306.

Lorenzen K. (1993) Assessment and management of south Asian small reservoir fisheries: a population dynamics approach. Report, MRAG Ltd., 120 pp.

McGurk M.D. (1986) Natural mortality of marine pelagic fish eggs and larvae: the role of spatial patchiness. Marine Ecology - Progress Series 34, 227-242.

MeGurk M.D. (1987) Natural mortality and spatial patchiness: a reply to Gulland. Marine Ecology -Progress Series 39, 201-206.

Peterson 1. \& Wroblewski LS. 1984. Mortality rate of fishes in the pelagic ecosystem. Canadian Journal of Fisheries and Aquatic Science 41, 1117-1120.

Shepherd J. G. (1987) Towards a method for short-term forecasting of catch rates based on length compositions. In: D. Pauly \& G.P. Morgan (eds). Length-Based Methods in Fisheries Research. Manila: ICLARM, pp. 113-120.

Walters C.J. (1986) Adaptive Management of Renewable Resources. New York: Mactnillan, 374 pp.

\section{Appendix: The population model}

\section{Population and catch equations}

The fish population is divided into length groups, so that the population at time $t$ is represented by a vector $\mathrm{n}$, of numbers-at-length. The population equation computes this vector for time $\mathrm{t}+\mathrm{dt}$ :

$$
n_{t+d t}=G_{B} S\left(n_{t}+s t_{t}\right)
$$

where $G_{B}$ is the density-dependent growth projection matrix, $\mathrm{S}$ is the survival matrix, and $\mathrm{st}_{\mathrm{t}}$ is the vector of fish stocked-at-length at time t. 
The corresponding catch equation gives the vector $\mathrm{c}$, of catch-at-length obtained during the time interval from $t$ to $t+d t$ :

$$
c_{t}=G_{B} H\left(n_{t}+s t_{t}\right)
$$

where $\mathrm{H}$ is the harvesting matrix.

\section{Grovvth projection matrix}

The element $g_{i j}$ of the growth projection matrix is the proportion of fish in the initial length group $j$ that contributes to the final group $i$. The matrix is constructed using the method of Shepherd (1987). At time $t+d t$, the length interval $\left[L_{i}, L_{i+}\right]$ contains the surviving individuals that at time $t$ were within the interval $\left[L_{i}, L_{i_{+}}\right]$defined by the back-calculated lengths of the current bounds. The back-calculated length $L_{i}^{\prime}$ of bound $L_{i}$ is given by

$$
L^{\prime}{ }_{i}=\left\{L_{i}-L_{x B}[1-\exp (-\mathrm{K} d t)]\right\} / \exp (-\mathrm{K} d t)
$$

where $L_{\infty \circ B}$ is the $L_{\infty}$ at biomass B obtained from Eq. (1), and $\mathrm{K}$ is the von Bertalanffy parameter K.

Once the former bounds of the length groups have been calculated, the elements $g_{i j}$ of the growth projection matrix are determined by the following algorithm:

$$
\begin{array}{ll}
\text { if } L_{\mathrm{j}}<L_{i}{ }_{i}<L_{j+1} & \text { then } g_{i j}=\left(L_{j+1}-L_{i}{ }_{i}\right) / d L \\
\text { if } L_{\mathrm{j}}<L^{\prime}{ }_{i+1}<L_{j+1} & \text { then } g_{i j}=\left(L_{i+1}-L_{j}\right) / d L \\
\text { if } L_{i}^{\prime}<L_{j} \text { and } L_{j+1}<L_{i+1}^{\prime} & \text { then } g_{i j}=1.0 \\
\text { if } L^{\prime}{ }_{i+1}<L_{j} & \text { then } g_{i j}=0 \\
\text { if } L_{i}^{\prime}>L_{j+1} & \text { then } g_{i j}=0 \\
\text { if } L^{\prime}{ }_{m}<L_{j} & \text { then } g_{m j}=1.0
\end{array}
$$

The highest length class $\mathrm{n}_{\mathrm{m}, \mathrm{t}}$ is defined as a plus group, it contains all individuals larger than the lower length bound of the group.

\section{Survival matrix}

The elements $s_{j j}$ of the diagonal survival matrix are the proportions of fish in length class $j$ at time $t$ survive to time $t+d t$. Survival $s_{j j}$ is a function of the size-dependent natural mortality rate $M_{j}$ (Eq. 2), fishing mortality rate $\mathrm{F}$, and gear selectivity $v_{j}$ :

$$
s_{j j}=\exp -\left(M_{j}+F v_{j}\right) d t
$$

\section{Harvesting matrix}

The elements $h_{j j}$ of the diagonal harvesting matrix are the proportions of fish in length class $j$ that are harvested during the time interval $[t, t+d t]$ :

$$
h_{j j}=\left[F v_{j} /\left(M_{j}+F v_{j}\right)\right]\left[1-\exp \left(-\left(M_{j}+F v_{j}\right) d t\right)\right]
$$

